\title{
The Versatile Imaging Capabilities of Aberration-Corrected STEM
}

\author{
Jingyue (Jimmy) Liu
}

\author{
Departments of Physics, Arizona State University, Tempe, Arizona 85287, USA
}

The recent advancement in aberration-corrected electron microscopy, especially aberration-corrected STEM (AC-STEM), makes it possible to routinely extract atomic scale information of a variety of solid materials. With the availability of sub-angstrom size electron probes and the appropriate selection of detectors one can extract useful information on the atomic structure, chemical composition, oxidation state, and strain field of the sample of interest. The common choice of selecting high-angle scattered electrons for atomic number contrast (HAADF imaging mode) and the directly transmitted electrons for phase contrast (BF-STEM imaging mode) may pose challenges when non-ideal samples are encountered. Fortunately, many other imaging modes are available in STEM and can be utilized to provide unique, although selective, information. For example, the large-angle bright-field (LABF) imaging mode provides information complementary to those of the HAADF imaging mode, and configured detectors were proposed to manipulate the scattered signals for desired information [1-2]. The thin annular detector BF (TADBF), thin annular detector DF (TADDF), and the marginal DF-BF detector configurations all provide unique information of the sample by judiciously utilizing the variations in the electron scattering profiles of different materials [3-5]. For example, light-element materials supported on heavy-element materials, such as carbon on platinum, have been imaged [4-5]. The flexibility of incorporating different types of detectors in the STEM makes it a versatile tool.

The recent applications of annular BF (ABF) imaging of oxygen, lithium and even hydrogen atoms in crystalline materials have attracted broad interest [6-7] and may significantly expand the applications of AC-STEM techniques to the investigation of a plethora of materials systems. Multi-imaging modalities can be simultaneously accomplished in an AC-STEM and each imaging mode may provide useful or complementary information. Figure 1 shows an atomic resolution HAADF image of an $\mathrm{Fe}_{3} \mathrm{O}_{4}$ nanoparticle oriented close to the [110] zone axis, revealing the different types of Fe columns as well as the surface atomic arrangement of the Fe atoms. Figure $2 \mathrm{a}$ and $2 \mathrm{~b}$ show, respectively, a HAADF and a BF-STEM image of a $\mathrm{ZnO}$ nanoplate oriented close to the [0001] zone axis. Both images provide atomic scale information on the arrangement of the $\mathrm{Zn}$ atoms and the surface reconstruction. Figure 3 shows a low-angle ADF (LAADF), an $\mathrm{ABF}$ and a medium-angle $\mathrm{BF}$ (MABF) image of a faceted $\mathrm{ZnO}$ nanowire. Both the LAADF and the ABF images provide information on the oxygen atoms. The imaging contrast of these various imaging modes, however, depends on the sample thickness, lens defocus, etc.

\section{References:}

[1] JM. Cowley, Ultramicroscopy 49 (1993), p. 4.

[2] J. Liu and JM. Cowley, Ultramicroscopy 52 (1993), p. 335.

[3] JM. Cowley, MS. Hansen and SY. Wang, Ultramicroscopy 65 (1996), p. 18.

[4] JM. Cowley, VI. Merkulov and JS. Lannin, Ultramicroscopy 58 (1995), p. 61.

[5] RJ. Liu and JM. Cowley, Microscopy and Microanalysis 2 (1996), p. 9.

[6] SD. Findley et al., Appl. Phys. Lett. 95 (2009), 191913.

[7] R. Ishikawa et al., Nature Materials 10 (2011), p. 278. 
[8] This research was funded by Arizona State University. We gratefully acknowledge the use of facilities within the LeRoy Eyring Center for Solid State Science at Arizona State University.
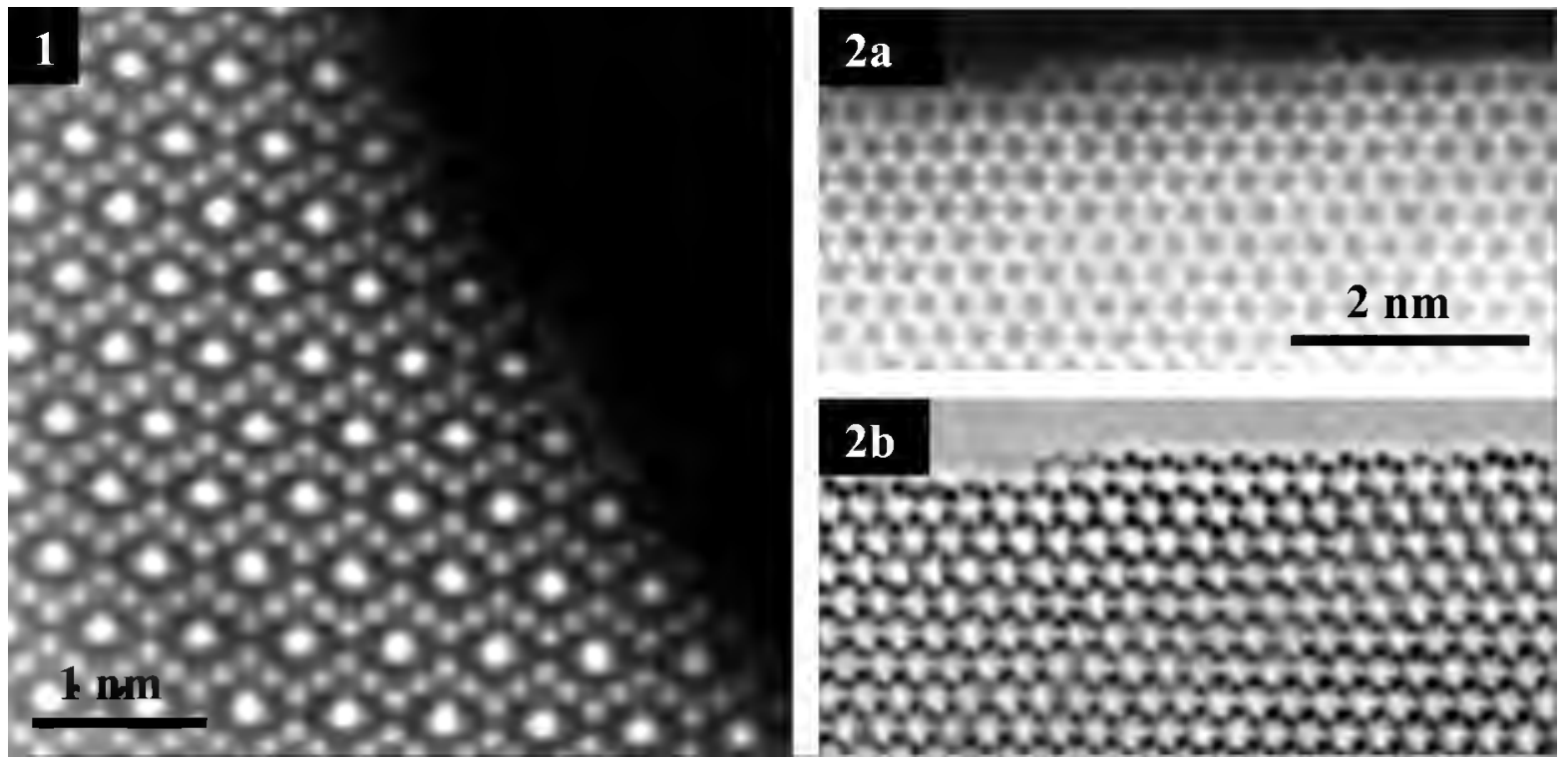

Figure 1. Sub-angstrom resolution image of an $\mathrm{Fe}_{3} \mathrm{O}_{4}$ nanoparticle, oriented close to the [110] zone axis, clearly reveals the two types of the Fe columns and the reconstruction of the surface $\mathrm{Fe}$ atoms.

Figure 2. HAADF (a) and BF-STEM (b) images of the edge of a hexagonal $\mathrm{ZnO}$ plate, oriented close to the $\mathrm{ZnO}[0001]$ zone axis. Both the atomic number contrast HAADF and the phase contrast BF-STEM images show the surface atomic arrangement of the $\mathrm{ZnO}$ nanoplates.

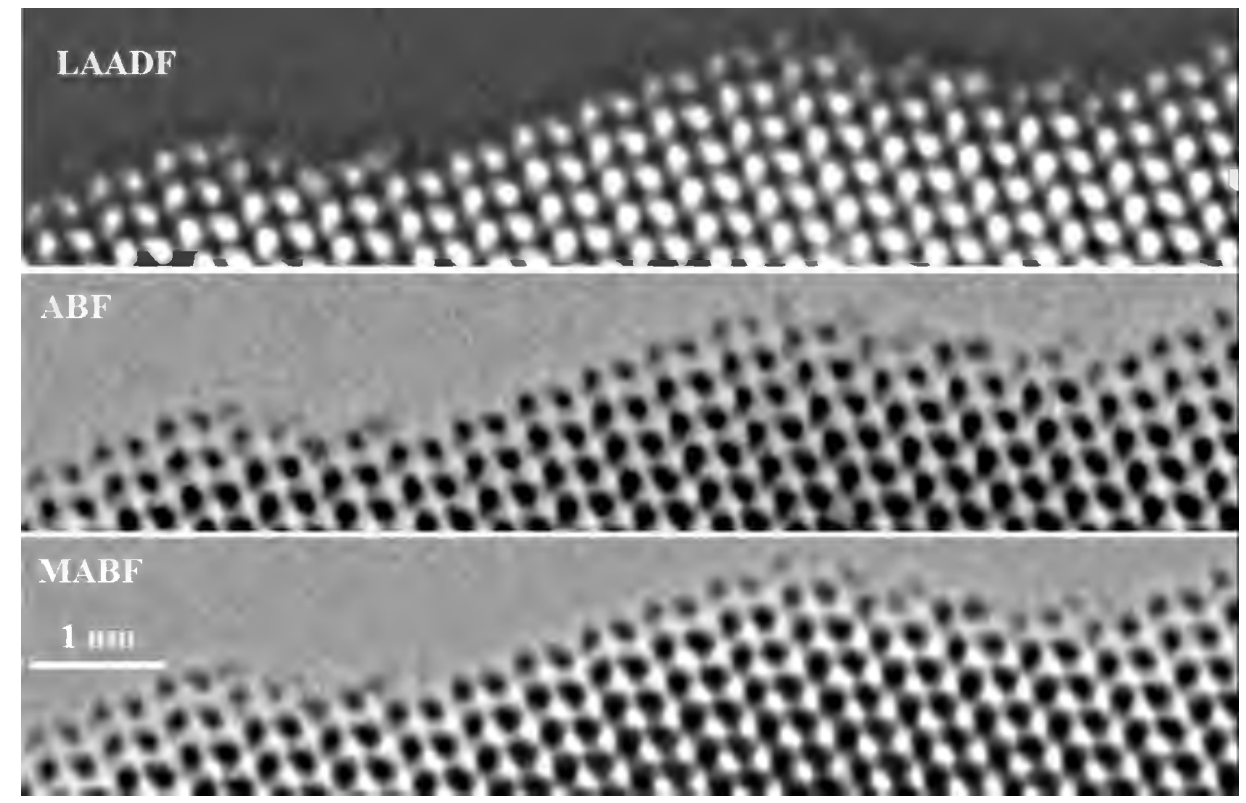

Figure 3. Low-angle $\mathrm{ADF}$ (LAADF), $\mathrm{ABF}$, and medium-angle $\mathrm{BF}(\mathrm{MABF})$ images of a faceted $\mathrm{ZnO}$ nanowire. The $\mathrm{ZnO}$ nanowire was oriented close to the [11-20] zone axis. Both the LAADF and the $\mathrm{ABF}$ images show the oxygen columns, especially at the thinner regions of the nanowire. Surface reconstructions along the different facets are also discernible. 